\title{
Terahertz Multilayer Thickness Measurements: Comparison of Optoelectronic Time and Frequency Domain Systems
}

\author{
Lars Liebermeister $^{1}$ (D) . Simon Nellen ${ }^{1}$ (D) Robert B. Kohlhaas $^{1}$ (iD) \\ Sebastian Lauck ${ }^{1} \cdot$ Milan Deumer $^{1}(\mathbb{D})$. Steffen Breuer ${ }^{1}(\mathbb{D}) \cdot$ Martin Schell $^{1,2}$. \\ Björn Globisch ${ }^{1,2}$ (1)
}

Received: 22 June 2021 / Accepted: 3 November 2021/Published online: 8 December 2021

(c) The Author(s) 2021

\begin{abstract}
We compare a state-of-the-art terahertz (THz) time domain spectroscopy (TDS) system and a novel optoelectronic frequency domain spectroscopy (FDS) system with respect to their performance in layer thickness measurements. We use equal sample sets, $\mathrm{THz}$ optics, and data evaluation methods for both spectrometers. On singlelayer and multi-layer dielectric samples, we found a standard deviation of thickness measurements below $0.2 \mu \mathrm{m}$ for TDS and below $0.5 \mu \mathrm{m}$ for FDS. This factor of approx. two between the accuracy of both systems reproduces well for all samples. Although the TDS system achieves higher accuracy, FDS systems can be a competitive alternative for two reasons. First, the architecture of an FDS system is essentially simpler, and thus the price can be much lower compared to TDS. Second, an accuracy below $1 \mu \mathrm{m}$ is sufficient for many real-world applications. Thus, this work may be a starting point for a comprehensive cross comparison of different terahertz systems developed for specific industrial applications.
\end{abstract}

\section{Introduction}

Terahertz (THz) spectroscopy is an interesting sensing technology for many applications in material and structural analysis, compound identification, and testing $[1,2]$. The measurement is non-contact and non-destructive, can easily handle airmaterial interfaces, and uses non-ionizing radiation. One of the key applications for $\mathrm{THz}$ spectroscopy is the thickness measurements of paint and coating layers. Until today, time domain spectroscopy (TDS) is almost exclusively used for this kind of

Lars Liebermeister

lars.liebermeister@hhi.fraunhofer.de

1 Fraunhofer Institute for Telecommunications, Heinrich Hertz Institute, Einsteinufer 37, 10587 Berlin, Germany

2 Institut Für Festkörperphysik, Technische Universität Berlin, Hardenbergstraße 36, EW 5-1, 10623 Berlin, Germany 
applications, as these systems are mature and commercially available. In addition, THz TDS offers high acquisition speed and high THz bandwidth. The latter enables thickness measurements of sub-mm dielectric layers [3-5] and the detection of defects in polymers, foams, and other non-conductive materials [6].

In contrast, frequency domain spectroscopy (FDS), which is based on the generation and coherent detection of continuous wave (cw) $\mathrm{THz}$ radiation, hitherto is barely used for non-destructive testing (NDT) [7, 8]. The inherently low measurement rate of most scientific FDS implementations and all commercial FDS products makes NDT with cw THz radiation highly inefficient. However, it was shown already in 2014 that THz FDS can be used for multilayer thickness measurements [9]. We have recently demonstrated a THz FDS system that allows acquisition rates of $200 \mathrm{~Hz}$, which is comparable to the speed of most commercial TDS systems. With this FDS system, a PET film with a thickness of $23 \mu \mathrm{m}$ could be measured with an accuracy better than $2 \%$, which corresponds to an uncertainty below $0.5 \mu \mathrm{m}$. This demonstrates that FDS can compete with TDS for accurate thickness measurements on thin dielectric layers [10]. Thereby, the main advantage of FDS compared to TDS is the simplicity of its system architecture. FDS systems are all-fiber coupled, and they do not require femtosecond optical pulses, moving optics, or complex phase locking electronics. This is why many industrial applications may benefit from using frequency domain spectroscopy instead of TDS. To date, there is no detailed comparison between the two methods in terms of measurement accuracy, measurement time, and reproducibility.

In this paper, we present the first direct comparison of a state-of-the-art TDS and optoelectronic FDS system for layer thickness measurements in reflection geometry. Both TDS and FDS measurements were performed on the same samples, namely PET films with thicknesses between 23 and $350 \mu \mathrm{m}$, a Si wafer with a thickness of $350 \mu \mathrm{m}$, and a ceramic coated with spray paint on both sides. Based on this, we compare the ability of the two systems to achieve the same results in consecutive measurements. Thereby, we analyze the effects of instrument noise, dynamic range, and bandwidth of the respective system on thickness determination. As the central figure of merit, we investigated the standard deviation of consecutive measurements at the same position on the sample. We found that the standard deviation of both TDS and FDS is always lower than $2 \%$.

This paper is organized as follows: In Section 2 we describe in detail the TDS and FDS system used for the comparison. Section 3 explains the experimental setup, data evaluation, and thickness determination. The measurement results are presented and discussed in Section 4 before we summarize our results in Section 5.

\section{TDS and FDS System}

The TDS measurements in this comparison were done with a commercial state-ofthe-art system, the TeraFlash pro system from TOPTICA Photonics AG [3]. A functional diagram of its optical components is shown in Fig. 1a. The system uses a femtosecond fiber laser centered at $1560 \mathrm{~nm}$, which generates pulses with a duration of $100 \mathrm{fs}$ at a repetition rate of $100 \mathrm{MHz}$. A voice-coil driven-optical delay allows for 

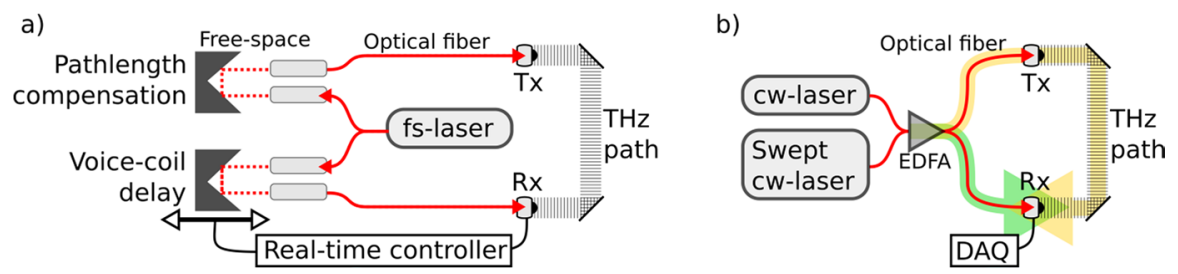

Fig. 1 Schematics of the two optoelectronic THz spectrometers used in this paper. (a) The TDS system compromises a pulsed fiber laser (fs-laser), a fast (voice-coil delay) and a slow (path length compensation) free-space optical delay, photoconductive emitter (Tx) and receiver (Rx), and a real-time controller to drive the voice-coil and acquire the data. All components are fiber-coupled. (b) The FDS system compromises a fixed-frequency (cw-laser) and a swept laser (Swept cw-laser), an erbium-doped fiber amplifier (EDFA), a PIN-photodiode emitter (Tx), and a photoconductive receiver (Rx) as well as a data acquisition unit (DAQ). The Tx- and Rx paths are indicated with yellow- and green-shaded arrows, respectively. The different path length in the Tx and $\mathrm{Rx}$ arm of the spectrometer are a prerequisite for the optoelectronic FMCW technique [10]

time-domain sampling with update rates up to $100 \mathrm{~Hz}$. An additional optical delay line in the receiver arm of the spectrometer allows for compensating a wide range of $\mathrm{THz}$ free space path lengths. Fiber-coupled InGaAs-based photoconductive modules are used as emitter (Tx) and receiver ( $\mathrm{Rx})$, respectively. The photoconductive material of the receiver is rhodium-doped InGaAs (InGaAs:Rh) [11, 12]. The Tx uses iron-doped InGaAs (InGaAs:Fe) [13]. In this configuration, the spectral maximum of the system is centered at $1 \mathrm{THz}$, and the peak dynamic range reaches $70 \mathrm{~dB}$ in single shot (66 ms measurement time) and exceeds $95 \mathrm{~dB}$ for 1000 averages (see Fig. 2 a). The total available bandwidth is more than $6 \mathrm{THz}$ and the spectral resolution can be as low as $1 \mathrm{GHz}$. All measurements presented here were acquired with a scan range of $70 \mathrm{ps}$, corresponding to a spectral resolution of $14 \mathrm{GHz}$.

FDS measurements were performed with a recently published optoelectronic continuous-wave terahertz spectrometer [10]. The working principle of this system is based on frequency-modulated continuous-wave (FMCW), which is an
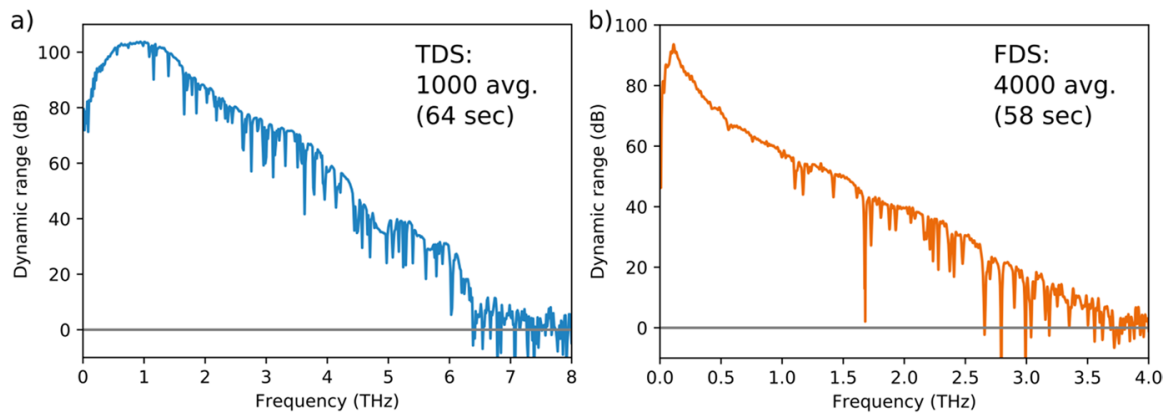

Fig. 2 Comparison of typical spectra recorded with the TDS (a) and FDS (b) systems. The dynamic range is plotted against frequency. Both records use $1 \mathrm{~min}$ of averaging. In contrast to the measurements on the sample, these signals are recorded in a transmission setup consisting of two off-axis parabolic mirrors, which is filled with air of ambient humidity 
established technique used in purely electronic THz systems [14]. A schematic diagram of the optoelectronic THz FDS system is shown in Fig. 1b. The spectrometer uses two fiber-coupled, continuous-wave semiconductor lasers emitting in the c-band (1530-1565 nm). The frequency of one of these lasers is swept periodically, while the emission frequency of the second laser stays fixed. The laser outputs are spatially overlapped in a $3 \mathrm{~dB}$ coupler, which generates an optical beat note. This beat note is amplified by an EDFA before being converted into $\mathrm{THz}$ radiation via photomixing in a waveguide-integrated PIN photodiode $[15,16]$. The same beat signal is also used to drive the coherent detection in the photoconductive $\operatorname{Rx}$ [15]. Tx and Rx are based on commercially available, fiber-coupled modules from TOPTICA Photonics AG. Further details on the THz FDS system can be found in the literature [10]. The most significant and fundamental difference of this FDS system compared to common setups with coherent detection of $\mathrm{cw} \mathrm{THz}$ signals is the method employed to obtain the phase. When no phase modulation is used, fringe-detection or Hilbert-transform [17] are common methods to extract the phase information. Alternatively, active phase modulation by a free-space optical delay line [18], a fiber stretcher [19], or an optical phase modulator [20,21] can be employed, which allows for amplitude and phase determination with a quadrature lock-in detector. The FDS system used here bases on an optoelectronic adaption of the FMCW technique [10], which results in passive phase modulation. The tunable cw-laser is frequency swept with more than $500 \mathrm{THz} / \mathrm{sec}$. In combination with a path length imbalance of $20 \mathrm{~cm}$ between the emitter and the receiver arm (indicated by shaded arrows), an intermediate frequency of $500 \mathrm{kHz}$ is generated in the photomixing receiver, which can be directly used for coherent detection with a software-based lock-in amplifier. This quadrature lock-in detection allows to detect amplitude and phase as a function of frequency. Note that this FDS system neither requires optomechanics nor free space optics nor electro-optic phase modulation. The $\mathrm{THz}$ amplitude spectrum acquired with the FDS system is depicted in Fig. 2 b. The spectral maximum is centered at $100 \mathrm{GHz}$ with a peak dynamic range exceeding $90 \mathrm{~dB}$ for 4000 averages. In a single shot measurement, which is acquired in $14 \mathrm{~ms}$, the dynamic range measures $60 \mathrm{~dB}$ with a $2 \mathrm{THz}$ bandwidth. With averaging, the peak dynamic range and bandwidth can reach $117 \mathrm{~dB}$ and $4 \mathrm{THz}$, respectively [10]. Figure 2 compares the THz spectra acquired with the TDS and the FDS system for a measurement time around $60 \mathrm{~s}$.

\section{THz-Setup and Measurement Procedure}

This section covers the experimental setup, the set of samples, the data preparation, and the algorithm for thickness determination.

\subsection{Experimental Setup}

All measurements were carried out in reflection geometry, which is the most industrially relevant setup. It requires only single-side access to the sample under test, and reflection measurements can be performed independent of the substrate. 
A schematic diagram and photograph of the $\mathrm{THz}$ reflection head used for TDS and FDS measurements are depicted in Fig. 3. Since the mechanical dimensions and beam profile of pulsed and cw emitter and receiver modules are almost identical, the same THz optical beam path is used for both systems. A $90^{\circ}$ off-axis parabolic mirror with 2 inch focal length collimates the $\mathrm{THz}$ beam of the emitter and another $90^{\circ}$ off-axis parabolic mirror with 4 inch focal length focuses the beam on the sample surface under an angle of $8^{\circ}$. The reflected $\mathrm{THz}$ beam is collected and focused on the detector by an equal configuration of mirrors. The diameter of all parabolic mirrors measures 1 inch, which allows for constructing a comparably compact reflection head. The resulting measurement spot is about $1 \mathrm{~mm}$ in diameter and the THz-beam is s-polarized with respect to the plane of incidence. The beam path is oriented upwards (see Fig. 3), which allows for placing the samples reproducibly in the $\mathrm{THz}$ focus. In order to suppress $\mathrm{THz}$ absorption from water vapor, the reflection head is encapsulated (housing in Fig. 3) and purged with nitrogen (see photograph in Fig. 3)). Between background, reference, and sample measurement, the alignment of the reflection head was kept unchanged.

The placement of the sample in the THz-beam path as well as the alignment is a common cause of variations of the measurement result. This effect can even dominate the uncertainty of thickness measurements in industrial applications. However, these variations are mainly related to the geometry of the samples, the sample holder, the design of the $\mathrm{THz}$ optics, and environmental conditions. Therefore, it is not an effect caused by the THz system used. In our comparison, we tried to minimize the influence of positioning errors and sample inhomogeneity by focusing our analysis on the reproducibility of consecutive measurements taken on the same position on the sample.

Fig. 3 Schematic and photograph of the reflection head used for non-contact layer thickness measurements. The reflection head was identical for TDS and FDS measurements. The diameter of the parabolic mirrors measures 1". In this view, $\mathrm{THz}$ transmitters and receivers and their respective beam paths are arranged one behind the other. Both beam paths hit the sample at the same spot and under an angle of $8^{\circ}$. Tx and Rx have a distance of $5 \mathrm{~mm}$. The housing is purged with nitrogen to avoid detrimental effects from water vapor absorption

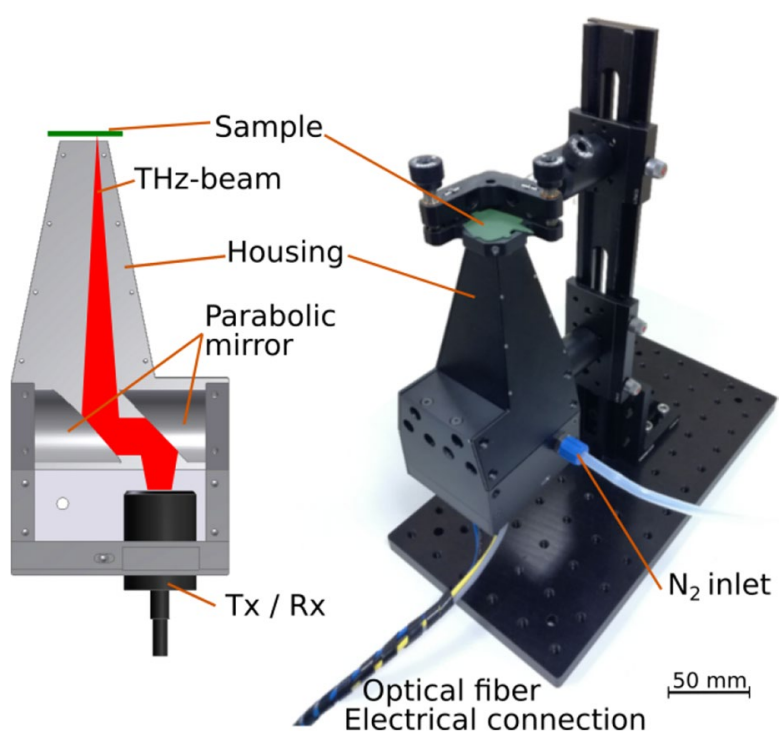

Springer 


\subsection{Measurement Conditions}

For the comparison presented in this paper, the FDS and TDS system were driven under almost identical conditions. It is well known that bandwidth and dynamic range increase significantly with a higher number of averages, i.e., longer measurement time. In general, the central limit theorem states that the dynamic range increases by $10 \mathrm{~dB}$ per tenfold increase in acquisition time, assuming a random noise background [10]. Therefore, the measurement time is a crucial parameter in the system comparison, which can be adjusted by applying a different number of averages for TDS and FDS. Figure 4 compares the THz spectra acquired with the two systems in reflection geometry. Here, the terahertz reflection head shown in Fig. 3 and a metal reflector were used. The signal acquisition time was almost identical for both systems. The TDS data was acquired by averaging two subsequent pulse traces with total acquisition time of $127 \mathrm{~ms}$. The peak dynamic range was $>70 \mathrm{~dB}$ at $1 \mathrm{THz}$ and the bandwidth exceeded $5 \mathrm{THz}$. The FDS data was acquired with ten averages, resulting in a total measurement time of $147 \mathrm{~ms}$. Under these conditions, the peak dynamic range was $>50 \mathrm{~dB}$ at $100 \mathrm{GHz}$ and the total bandwidth was $2 \mathrm{THz}$. Hence, the bandwidth of the TDS system is approximately twice as large as the bandwidth of the FDS system. In contrast, the frequency resolution of the FDS system measures $1 \mathrm{GHz}$, whereas the frequency resolution in TDS is $14 \mathrm{GHz}$ only. Before the results are being discussed and compared in Section 4 , the procedure of data analysis is described in the next paragraph.

\subsection{Data Preparation}

For a direct comparison of $\mathrm{THz}$ data acquired with the two different systems, the data post-processing and thickness evaluation methods have to be identical for both systems. Figure 5 depicts this unified post-processing procedure. For TDS, a fast Fourier transform (FFT) converts the measured pulse trace into a complex spectrum that can be represented in the frequency domain either by amplitude
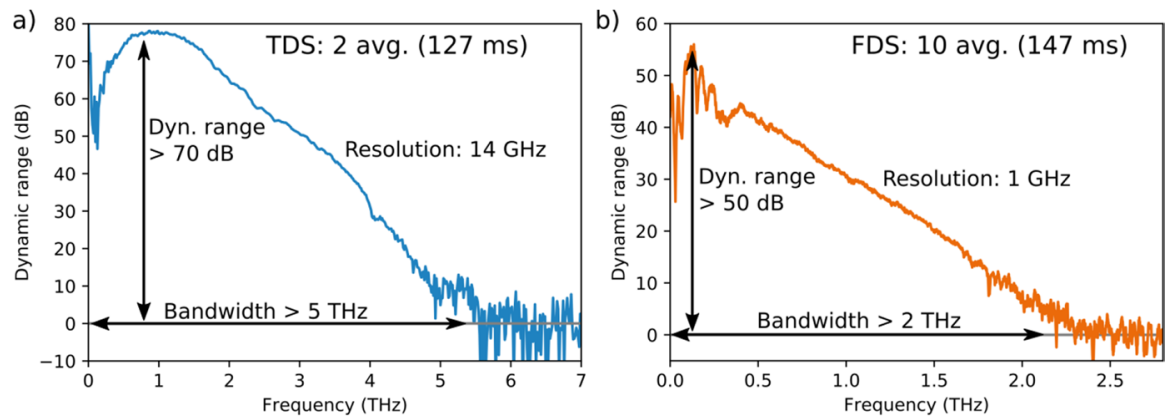

Fig. 4 Spectra recorded with the TDS (a) and FDS (b) system using the terahertz reflection head shown in Fig. 3 and a metal reflector. The spectra are recorded using the same optics, alignment procedure, acquisition and averaging parameters, and environmental conditions as the sample measurement in this work. The right spectrum is smoothed with a $10 \mathrm{GHz}$ window 


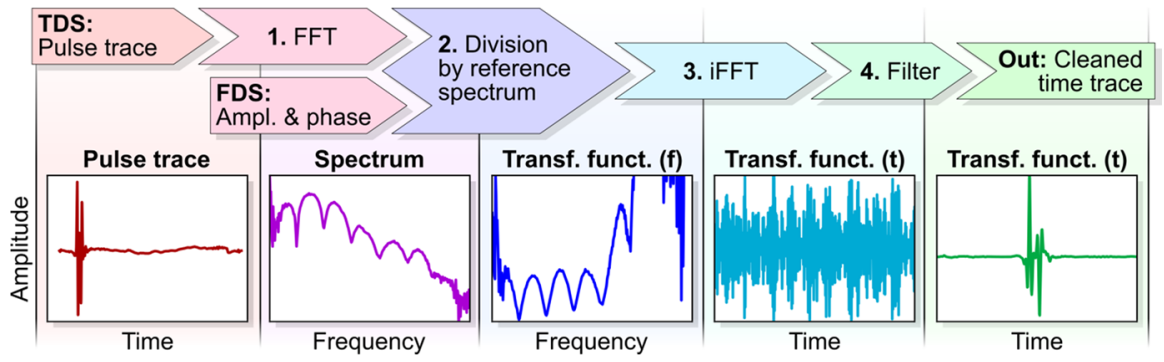

Fig. 5 Preparation procedure for the TDS- and the FDS-data. A representative sketch of the data after each step is shown at the bottom. The procedure corresponds to a deconvolution of the TDS pulse trace. Abbreviations: FFT, fast Fourier transform; Ampl., amplitude; iFFT, inverse fast Fourier transform

and phase or by a complex number. These spectra have the same data structure as the spectra acquired with the FDS system. Hence, all following data processing steps are identical for both data sets. First, the complex transfer function of the sample is determined by dividing the spectra of the sample measurement by a reference spectrum recorded with a metal reflector (see Fig. 4). Ideally, this transfer function contains the spectral response of the sample only. Spectral features that stem from the respective spectroscopic system and the setup are thereby eliminated. Next, an inverse FFT (iFFT) transforms the transfer function into the timedomain, and subsequently, frequency filters are applied to the time-domain signal to remove noise and artefacts. In the resulting time trace, each dielectric interface with an increase or decrease in the refractive index appears as a positive or negative peak, respectively (see Fig. 6). Note that these processing steps correspond to a deconvolution of the TDS pulse trace. The frequency-filters are of Butterworthtype and applied forward-backward so that a flat phase response is ensured. Initially, the filter parameters are optimized manually to suppress background noise and ringing while keeping the closest peaks well separated. The low-pass $3 \mathrm{~dB}$ frequency was found to be critical for the thickness evaluation, and therefore a value sweep was performed to find an optimal value (see Section 4 for details). After this optimization, the filter parameters were kept constant for all measurements and identical for TDS and FDS.

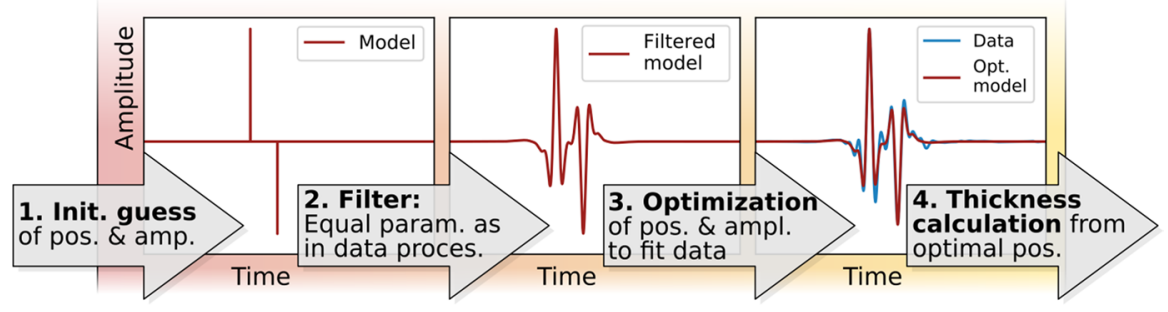

Fig. 6 Thickness determination procedure 


\subsection{Thickness Determination}

In the processed time trace data, thick layers can be clearly identified by wellseparated peaks, which enable thickness determination via basic peak-finding algorithms. However, this simple procedure fails for thin layers where individual peaks overlap. Therefore, we use a model-based approach to thickness determination. Figure 6 is a guide through the procedure:

1. Ideally sharp interfaces are assumed, which correspond to measurements with infinite bandwidth and dynamic range. Under this assumption, each interface is represented by a $\delta$-peak with a scaling factor for its amplitude and its position in the time domain. A model time-trace is generated from a series of $\delta$-peaks with initially guessed positions and amplitudes.

2. Frequency filters are applied to the model time-trace using the same coefficients as they were applied to the measured data.

3. This filtered model is compared to the recorded transfer function in the time domain. Steps 1 and 2 are repeated with varying position and amplitude of the initial $\delta$-peaks. Thereby, the model is optimized to fit the recorded data. Commonly, a least squares method is used for such an optimization processes. However, this approach does not converge well for a time trace containing a few peaks only. In the least squares method differences between model and data have the same weight for all data points. In our case most of the data points of the time-trace are close to zero and carry no information about the thickness. In order to increase the weight of the peaks, we multiply the square of the model function with the square of the difference between model and data. The result is then used as the penalty function, which is to be minimized.

4. We determine the layer thickness from the positions of the $\delta$-peaks of the optimal model. The time difference between two adjacent peaks is multiplied by the literature values of the refractive indices of the respective material.

Note that this procedure is similar to the frequently-used transfer matrix method. However, it is essentially simpler since refractive index and absorption coefficient of the material are not optimized. This simplification makes the procedure more robust against inaccurate alignment, surface roughness, and environmental influences. A possible disadvantage of this method is the detailed knowledge required about the sample under test, which includes the material of the different layers, the number of interfaces, and the $\mathrm{THz}$ refractive indices. However, for most industrial applications, this information is either already available or it can be determined in off-line characterization measurements.

The measurements with TDS and FDS were conducted using two reflection heads of equal design; therefore the data was not necessarily recorded on precisely the same spot of the sample. Inhomogeneities in material composition, refractive index, and local thickness can lead to differences in the thickness values obtained with the two systems. Hence, we choose the relative uncertainty of consecutive measurements as the figure of merit for the comparison of TDS and FDS measurements. 
Each measurement was repeated 50 times on the same position without movement of the sample. Afterward, we determined the average thickness value and the standard deviation. The latter is a measure for the repeatability of the thickness measurement and indicated as error bars where applicable. In general, absolute accuracy of a measurement is a matter of calibration, which corrects for systematic errors. However, the feasibility of the technology depends on the capability to obtain the same results in repeated measurements. Therefore, we use the standard deviation of subsequent measurements as central figure of merit. This value is independent of both, the precise calibration of the system and the knowledge of the exact material parameters. Thereby, the systems can be compared directly without considering any calibration procedures.

\section{Results}

In this paper, we measured the same set of single and multi-layer dielectric samples with both the TDS and the FDS system. The single layer samples were PET foils (polyethylene terephthalate) with nominal thicknesses of $23 \mu \mathrm{m}, 125 \mu \mathrm{m}, 250 \mu \mathrm{m}$, and $350 \mu \mathrm{m}$ and two Kapton foils (PI, Polyimide) with thicknesses of $50 \mu \mathrm{m}$ and $75 \mu \mathrm{m}$. The multi-layer sample consists of a ceramic substrate with green spray paint on both sides. All samples were placed on the sample holder shown in Fig. 3 and measured under nitrogen atmosphere. The thicknesses were determined by using literature values for the refractive indices, which are $n_{\mathrm{Kapton}}=1.87, n_{\mathrm{PET}}=1.75$ [22], $n_{\text {paint }}=1.5$, and $n_{A L 2 O 3}=3$ [23]. For simplicity, this evaluation does not account for frequency-dependent refractive indices. The resulting small systematic error in the absolute thickness values affects both systems similarly. Note that the thickness evaluation procedure does not evaluate the reflection intensity at the interfaces; therefore, absorption coefficients are not included.

Figure 7(a) shows the measured thickness as a function of the nominal thickness for the six single layer samples. The diagonal, dashed gray line indicates perfect
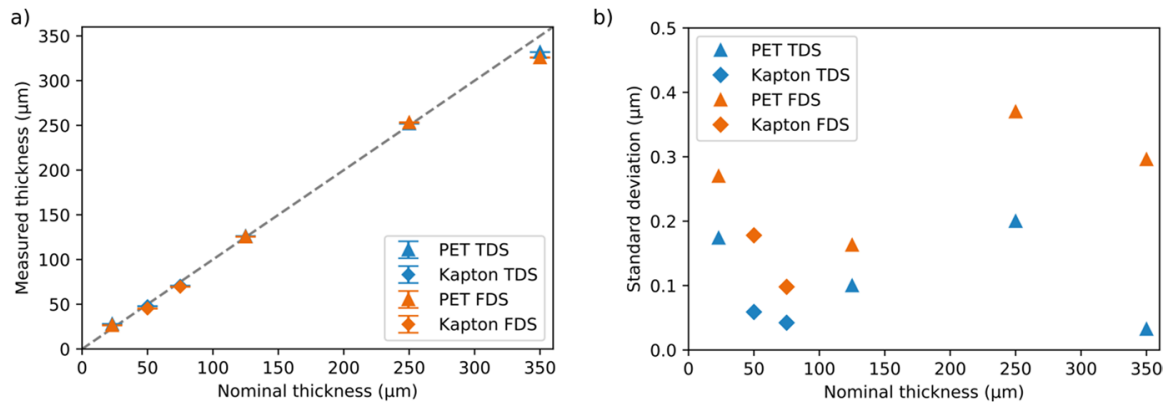

Fig. 7 (a) Measured thickness as a function of nominal thickness for the set of single layer dielectric foils. Blue (orange) symbols correspond to FDS (TDS) measurements. The dashed gray line is a guide to the eye showing perfect agreement of nominal and measured thickness. (b) Standard deviation as a function of the nominal thickness for 50 consecutive measurements on the single layer PET and Kapton foils shown in (a) 
agreement between nominal and measured thickness. Blue/orange symbols denote TDS/FDS measurements, respectively. Above all, the measured values are very close to the expected nominal thickness. The highest deviation can be observed for the $350 \mu \mathrm{m}$-PET foil. As both FDS and TDS measure a lower layer thickness than expected, we attribute this discrepancy to a slightly thinner or not perfectly homogeneous sample. However, both measurement techniques deliver layer thicknesses with small standard deviations in consecutive measurements (indicated as error bars) for all single-layer samples. Hence, the thickness can be measured in a reproducible way with both techniques.

As the error bars in Fig. 7 (a) are very small, Fig. 7 (b) compares the standard deviations of all samples as a function of the nominal thickness. In addition, Table 1 summarizes the results. The standard deviation of the thicknesses measured with TDS ranges from $0.04 \mu \mathrm{m}$ to $0.20 \mu \mathrm{m}$, whereas the standard deviations of the FDS measurements are $0.10 \mu \mathrm{m}$ to- $0.37 \mu \mathrm{m}$. Hence, the accuracy of the TDS system is by a factor of approx. two higher compared to the FDS measurements. However, all uncertainties are in the sub-micron regime, which demonstrates that both systems are well suited for precise layer thickness measurements. Even for the thinnest sample, the standard deviation is not higher than $1 \%$ of the nominal sample thickness. In particular, this shows that the single layer PET foil with $23 \mu \mathrm{m}$ in thickness is not yet below the resolution limit of the FDS system. This underlines the quality of the signal acquired with this system as well as the efficiency of the thickness evaluation procedure.

As mentioned before, the cutoff frequencies of the low- and high-pass filters, which are applied to the data and the model function in the time domain, affect the reproducibility of the thickness determination. Here, we demonstrate the effect exemplarily for the low-pass filter. For the high-pass filter, the arguments are analogous. If the cut-off frequency of the low-pass filter was too small, a significant amount of the signal would be cut out. This would reduce the signal strength and lead to overlapping peaks at thin interfaces, which increases the uncertainty of the thickness determination. If the cut-off frequency of the low-pass filter was too high, the noise in the time trace would increase, which again increases the standard deviation. This effect can be observed in Fig. 8, where the standard deviation of 10

Table 1 Layer thicknesses with absolute and relative standard deviations obtained with TDS and FDS measurements on single layer PET and Kapton foils

\begin{tabular}{|c|c|c|c|c|c|c|c|}
\hline \multirow[b]{2}{*}{ Material } & \multirow[b]{2}{*}{$\begin{array}{l}\text { Nominal } \\
\text { thickness } \\
(\mu \mathrm{m})\end{array}$} & \multicolumn{3}{|l|}{ TDS } & \multicolumn{3}{|l|}{ FDS } \\
\hline & & $\begin{array}{l}\text { Thickness } \\
(\mu \mathrm{m})\end{array}$ & $\begin{array}{l}\text { Std. Dev. } \\
(\mu \mathrm{m})\end{array}$ & $\begin{array}{l}\text { Rel. Std. } \\
\text { Dev. (\%) }\end{array}$ & $\begin{array}{l}\text { Thickness } \\
(\mu \mathrm{m})\end{array}$ & $\begin{array}{l}\text { Std. Dev. } \\
(\mu \mathrm{m})\end{array}$ & $\begin{array}{l}\text { Rel. Std. } \\
\text { Dev. (\%) }\end{array}$ \\
\hline PET & 23 & 27.97 & 0.17 & 0.61 & 26.50 & 0.27 & 1.02 \\
\hline Kapton & 50 & 47.86 & 0.06 & 0.12 & 45.74 & 0.18 & 0.40 \\
\hline Kapton & 75 & 70.75 & 0.04 & 0.06 & 70.31 & 0.10 & 0.14 \\
\hline PET & 125 & 126.20 & 0.10 & 0.08 & 125.50 & 0.16 & 0.13 \\
\hline PET & 250 & 252.00 & 0.20 & 0.08 & 253.20 & 0.37 & 0.15 \\
\hline PET & 350 & 331.85 & 0.03 & 0.01 & 325.80 & 0.30 & 0.09 \\
\hline
\end{tabular}


Fig. 8 Standard deviation of the thickness measurements on a $125 \mu \mathrm{m}$ PET foil as a function of the cutoff frequency of the low-pass filter used in the data preparation procedure (see Fig. 5). Orange (blue) symbols correspond to FDS (TDS) measurements, respectively. Open symbols denote the cutoff frequencies used for the results shown in Fig. 7

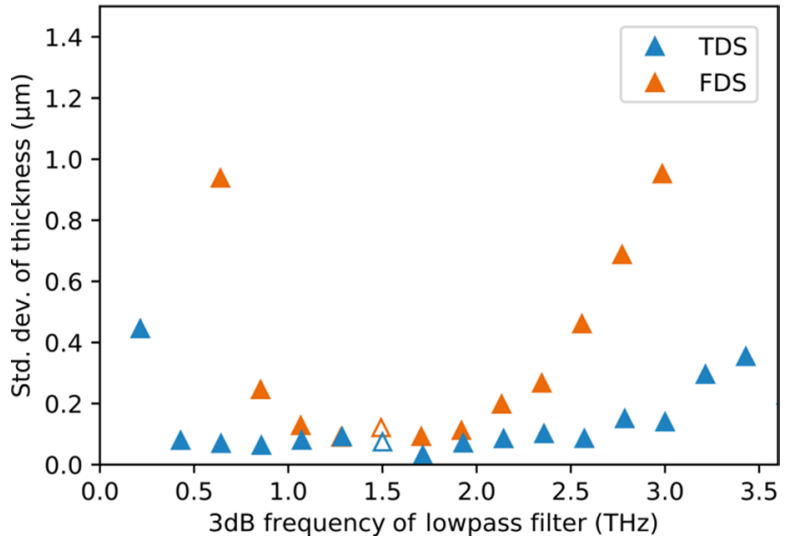

thickness measurements on a $125 \mu \mathrm{m}$ PET foil is plotted as a function of the cutoff frequency of the low-pass filter between $0.2 \mathrm{THz}$ and $3.5 \mathrm{THz}$. Blue/orange symbols correspond to TDS/FDS measurements, respectively. The open symbols denote the values used for the results shown in Fig. 7. The standard deviation for both systems shows a broad minimum, spanning from $0.5 \mathrm{THz}$ to $3.0 \mathrm{THz}$ for TDS and from 1 to $2 \mathrm{THz}$ for FDS. Within these frequency ranges, the standard deviation is well below $0.2 \mu \mathrm{m}$. Hence, the overall higher bandwidth and dynamic range of the TDS system leads to a larger interval of possible cutoff frequencies. However, these results also show that the cutoff frequency used in the previous single layer measurements (open symbols) did not artificially reduce the accuracy of the TDS system.

Finally, we compared the TDS and FDS system in thickness measurements on a tri-layer sample. The sample consists of a ceramic substrate (Al2O3) with a nominal thickness of $640 \mu \mathrm{m}$ and layers of green spray paint of different thickness on the front and on the backside. The experimental setup and the data evaluation was identical to the single layer samples. The filters used in the data evaluation were a first order high-pass with a $3 \mathrm{~dB}$ cut-off frequency at $0.22 \mathrm{THz}$ and a fourth order low-pass with a $3 \mathrm{~dB}$ cut-off frequency at $1.72 \mathrm{THz}$. Figure 9 shows the normalized transfer function of the TDS (blue) and the FDS (orange) measurement. The ceramic substrate and the two paint layers are highlighted by gray and green background colors, respectively. The different material boundaries can be identified clearly by their corresponding peaks in the transfer function. Note that differences between TDS and FDS measurement are small and almost invisible in this figure. The calculated thicknesses and standard deviations of 50 consecutive measurements are summarized in Table 2.

Similar to the measurements on the single layer samples, the absolute values of the layers differ slightly between the measurements with the two systems. This can be explained by different measurement positions on the sample. More interestingly, the standard deviation of subsequent measurements is as low as $0.2 \mu \mathrm{m}$ for the TDS and $0.5 \mu \mathrm{m}$ for the FDS system. Similar to the single layer samples, the standard deviation of the FDS system is about twice as large as the standard deviation of TDS. Nevertheless, the uncertainties of the measurements are well below $1 \mu \mathrm{m}$ for both systems. Note 


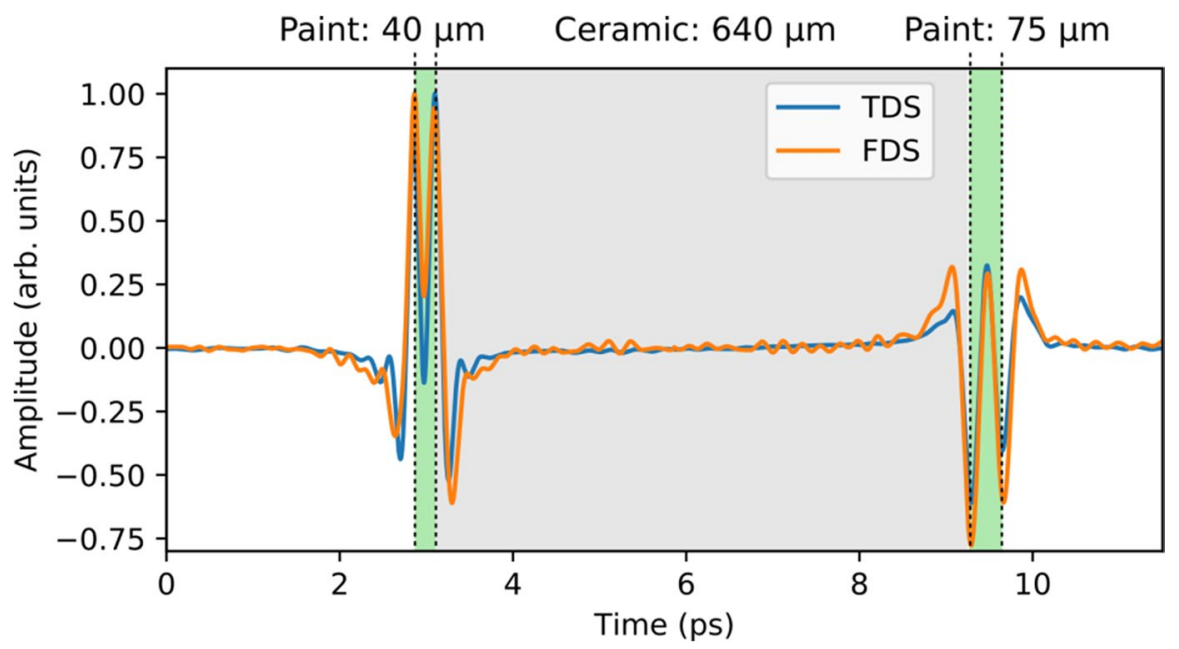

Fig. 9 Processed transfer function of the TDS (blue) and FDS (orange) measurement on a tri-layer sample in the time domain. The sample consists of a ceramic substrate with a nominal thickness of $640 \mu \mathrm{m}$ and green spray paint on the front and the backside. The peaks in the transfer function indicate the interfaces between different layers. Green and grey shaded areas indicate paint and ceramic, respectively

Table 2 Layer thicknesses of a tri-layer sample determined with the TDS and the FDS system

\begin{tabular}{|c|c|c|c|c|c|c|}
\hline \multirow[b]{2}{*}{ Material } & \multicolumn{3}{|l|}{ TDS } & \multicolumn{3}{|l|}{ FDS } \\
\hline & $\begin{array}{l}\text { Thickness } \\
(\mu \mathrm{m})\end{array}$ & Std. Dev. $(\mu \mathrm{m})$ & $\begin{array}{l}\text { Rel. Std. } \\
\text { Dev. (\%) }\end{array}$ & $\begin{array}{l}\text { Thickness } \\
(\mu \mathrm{m})\end{array}$ & Std. Dev. $(\mu \mathrm{m})$ & $\begin{array}{l}\text { Rel. Std. } \\
\text { Dev. (\%) }\end{array}$ \\
\hline $\begin{array}{l}\text { Spray paint } \\
\text { (front) }\end{array}$ & 46.1 & 0.1 & 0.2 & 44.3 & 0.2 & 0.4 \\
\hline $\begin{array}{l}\text { Ceramic sub- } \\
\text { strate }\end{array}$ & 619.4 & 0.1 & 0.02 & 616.5 & 0.2 & 0.04 \\
\hline $\begin{array}{l}\text { Spray paint } \\
\text { (back) }\end{array}$ & 72.0 & 0.2 & 0.3 & 72.8 & 0.5 & 0.7 \\
\hline
\end{tabular}

that the standard deviation of the first and the second layer is essentially lower than the value for the backside paint layer. This effect can be explained by the lower signal strength from the backside, which is caused by $\mathrm{THz}$ absorption in the material as well as reflection losses on previous interfaces (see Fig. 9).

\section{Summary and Outlook}

In this paper, we compared thickness measurement results on single and multilayer dielectric samples obtained with a commercially available THz TDS system and a novel optoelectronic FDS system. The measurements were performed in reflection geometry on the same sample set for TDS and FDS, respectively. The central figure 
of merit for the comparison was the standard deviation of 50 consecutive measurements at the same position on the sample. This allows to minimize external error sources like the inhomogeneity of the sample or the positioning accuracy of the sample. Hence, this comparison focusses on inherent system properties like instrument noise, dynamic range, and effective bandwidth. For five single layer PET and Kapton foils with thicknesses between $23 \mu \mathrm{m}$ and $350 \mu \mathrm{m}$, we found standard deviations smaller than $0.4 \mu \mathrm{m}$ with FDS and below $0.2 \mu \mathrm{m}$ with TDS. Although the TDS system features a bandwidth of more than $5 \mathrm{THz}$ and a peak dynamic range of $70 \mathrm{~dB}$ at $1 \mathrm{THz}$, the accuracy of the thickness measurements was just a factor of two higher than the accuracy of the FDS system, which has a $2 \mathrm{THz}$ bandwidth and a $50 \mathrm{~dB}$ peak dynamic range at $100 \mathrm{GHz}$. In the thickness measurements on a trilayer sample, this tendency manifested itself in the same way: the accuracy of TDS measurements was again just a factor of two higher than the accuracy of the FDS measurement. This demonstrates that FDS systems with a comparably simple architecture can be a promising alternative to complex TDS systems for layer thickness measurements. Additional effects such as sample positioning and alignment reduce the accuracy and thereby decrease the advantage of the TDS system. In future work we aim to extend this cross comparison by including different system architectures like electronically controlled optical sampling (ECOPS), asynchronous optical sampling (ASOPS), quasi TDS (QTDS) and THz cross-correlation spectroscopy with a superluminescent diode. Additionally, more sophisticated algorithms and physical modelling are planned to be applied to the data, which is likely to provide even more accurate results. Furthermore, different approaches might turn out to be optimal for a cw or a TDS system.

Authors' Contributions Conceptualization, L.L.; validation, S.N, R.B.K, S.L., M.A, S.B. and B.G.; investigation, L.L.; data curation, L.L.; writing-original draft preparation, L.L. and B.G.; writing-review and editing, M.S. and B.G.; supervision, M.S. and B.G.; funding acquisition, B.G. and L.L. All authors have read and agreed to the published version of the manuscript.

Funding Open Access funding enabled and organized by Projekt DEAL. This research was funded in part by Bundesministerium für Bildung und Forschung (BMBF), grant VIP+TeraLayerII, number 03VP07261.

Availability of Data and Material The data that support the findings of this study are available from the authors on reasonable request.

\section{Declarations}

Conflict of Interest The authors declare no competing interests.

Open Access This article is licensed under a Creative Commons Attribution 4.0 International License, which permits use, sharing, adaptation, distribution and reproduction in any medium or format, as long as you give appropriate credit to the original author(s) and the source, provide a link to the Creative Commons licence, and indicate if changes were made. The images or other third party material in this article are included in the article's Creative Commons licence, unless indicated otherwise in a credit line to the material. If material is not included in the article's Creative Commons licence and your intended use is not permitted by statutory regulation or exceeds the permitted use, you will need to obtain permission 
directly from the copyright holder. To view a copy of this licence, visit http://creativecommons.org/licen ses/by/4.0/.

\section{References}

1. M. Naftaly, N. Vieweg, and A. Deninger, "Industrial applications of terahertz sensing: State of play," Sensors, vol. 19, no. 4203, p. 4203, 2019, https://doi.org/10.3390/s19194203.

2. S. S. Dhillon et al., "The 2017 terahertz science and technology roadmap," J. Phys. D. Appl. Phys., vol. 50, no. 4, p. 43001, Jan. 2017, https://doi.org/10.1088/1361-6463/50/4/043001.

3. N. Vieweg et al., "Terahertz-time domain spectrometer with $90 \mathrm{~dB}$ peak dynamic range," J. Infrared Millim. Terahz Waves, vol. 35, no. 10, pp. 823-832, 2014, https://doi.org/10.1007/s10762-014-0085-9.

4. R. J. B. Dietz et al., "All fiber-coupled THz-TDS system with kHz measurement rate based on electronically controlled optical sampling," Opt. Lett., vol. 39, no. 22, pp. 6482-6485, 2014, https://doi.org/10. 1364/OL.39.006482.

5. T. Yasui, E. Saneyoshi, and T. Araki, "Asynchronous optical sampling terahertz time-domain spectroscopy for ultrahigh spectral resolution and rapid data acquisition," Appl. Phys. Lett., vol. 87, no. 6, pp. 2-5, 2005, https://doi.org/10.1063/1.2008379.

6. R. Wilk, T. Hochrein, M. Koch, M. Mei, and R. Holzwarth, "OSCAT: Novel technique for time-resolved experiments without moveable optical delay lines," J. Infrared, Millimeter, Terahertz Waves, vol. 32, no. 5, pp. 596-602, 2011, https://doi.org/10.1007/s10762-010-9670-8.

7. S. Preu, G. H. Döhler, S. Malzer, L. J. Wang, and A. C. Gossard, "Tunable, continuous-wave Terahertz photomixer sources and applications," J. Appl. Phys., vol. 109, no. 061301, 2011, https://doi.org/10. $1063 / 1.3552291$.

8. A. Roggenbuck et al., "Coherent broadband continuous-wave terahertz spectroscopy on solid-state samples," New J. Phys., vol. 12, 2010, https://doi.org/10.1088/1367-2630/12/4/043017.

9. D. Stanze, B. Globisch, R. Dietz, and H. Roehle, "Multilayer Thickness Determination Using Continuous Wave THz Spectroscopy," IEEE Trans. Terahertz Sci. Technol., vol. 4, no. 6, pp. 696-701, 2014.

10. L. Liebermeister et al., "Optoelectronic frequency-modulated continuous-wave terahertz spectroscopy with 4 THz bandwidth," Nat. Commun., vol. 12, no. 2021, pp. 1-10, 2021, https://doi.org/10.1038/ s41467-021-21260-x.

11. R. B. Kohlhaas et al., "Photoconductive terahertz detectors with $105 \mathrm{~dB}$ peak dynamic range made of rhodium doped InGaAs," Appl. Phys. Lett., vol. 114, no. 221103, p. 221103, 2019, https://doi.org/10. $1063 / 1.5095714$.

12. R. B. Kohlhaas et al., "Rhodium doped InGaAs: A superior ultrafast photoconductor," Appl. Phys. Lett., vol. 112, no. 10, 2018, https://doi.org/10.1063/1.5016282.

13. B. Globisch et al., "Iron doped InGaAs: Competitive THz emitters and detectors fabricated from the same photoconductor," J. Appl. Phys., vol. 121, no. 053102, 2017, https://doi.org/10.1063/1.4975039.

14. N. S. Schreiner, W. Sauer-Greff, R. Urbansky, G. von Freymann, and F. Friederich, "Multilayer Thickness Measurements below the Rayleigh Limit Using FMCW Millimeter and Terahertz Waves," Sensors, vol. 19, no. 18, p. 3910, 2019, https://doi.org/10.3390/s19183910.

15. S. Nellen, B. Globisch, R. B. Kohlhaas, L. Liebermeister, and M. Schell, "Recent progress of continuous-wave terahertz systems for spectroscopy, non-destructive testing, and telecommunication," in Proc of SPIE OPTO, 2018, no. 10531, https://doi.org/10.1117/12.2290207.

16. S. Nellen et al., "Experimental Comparison of UTC- and PIN-Photodiodes for Continuous-Wave Terahertz Generation," J. Infrared, Millimeter, Terahertz Waves, 2020, https://doi.org/10.1007/ s10762-019-00638-5.

17. D. W. Vogt, M. Erkintalo, and R. Leonhardt, "Coherent Continuous Wave Terahertz Spectroscopy Using Hilbert Transform," J. Infrared, Millimeter, Terahertz Waves, pp. 524-534, 2019, https://doi.org/ 10.1007/s10762-019-00583-3.

18. K. H. Park et al., "Photonic devices for tunable continuous-wave terahertz generation and detection," Proc. SPIE, vol. 7601, no. March 2014, p. 898506, 2014, https://doi.org/10.1117/12.2045639.

19. A. Roggenbuck et al., "Using a fiber stretcher as a fast phase modulator in a continuous wave terahertz spectrometer," J. Opt. Soc. Am. B, vol. 29, no. 4, p. 614, 2012, https://doi.org/10.1364/JOSAB.29. 000614.

20. D. Stanze, T. Göbel, R. J. B. Dietz, B. Sartorius, and M. Schell, "High-speed coherent CW terahertz spectrometer," Electron. Lett., vol. 47, no. 23, p. 1292, 2011, https://doi.org/10.1049/el.2011.3004. 
21. L. Liebermeister, S. Nellen, R. Kohlhaas, S. Breuer, M. Schell, and B. Globisch, "Ultra-fast, HighBandwidth Coherent cw THz Spectrometer for Non-destructive Testing," J. Infrared, Millimeter, Terahertz, Waves, vol. 40, no. 3, pp. 288-296, Jan. 2019, https://doi.org/10.1007/s10762-018-0563-6.

22. Y. Jin, G. Kim, and S. Jeon, "Terahertz Dielectric Properties of Polymers," J. Korean Phys. Soc., vol. 49, no. 2, pp. 513-517, 2006, https://doi.org/10.1002/app.1986.070310522.

23. K. Z. Rajab et al., "Broadband dielectric characterization of aluminum oxide (A1 2O3)," J. Microelectron. Electron. Packag., vol. 5, no. 1, pp. 2-7, 2008, https://doi.org/10.4071/1551-4897-5.1.1.

Publisher's Note Springer Nature remains neutral with regard to jurisdictional claims in published maps and institutional affiliations. 